\title{
Beam Synthesis with Low-Bit Reflective Coding Metamaterial Antenna: Theoretical and Experimental Results
}

\author{
Qinhao Wu (D), Yongqiang Cheng, Xiang Li, and Hongqiang Wang \\ College of Electronic Science, National University of Defense Technology, No. 109, Deya Road, Changsha, China \\ Correspondence should be addressed to Qinhao Wu; qinhaowu@hotmail.com
}

Received 1 November 2017; Revised 12 January 2018; Accepted 4 February 2018; Published 26 March 2018

Academic Editor: Giuseppe Castaldi

Copyright (C) 2018 Qinhao Wu et al. This is an open access article distributed under the Creative Commons Attribution License, which permits unrestricted use, distribution, and reproduction in any medium, provided the original work is properly cited.

\begin{abstract}
Coding metamaterials are the new technology where the aperture coding provides the hardware foundation for the miniaturization of the devices. As a synthetic 2-D plane, metamaterial antennas are composed of subwavelength resonant particles. It can realize real-time control of electromagnetic wave and build multifunction radar array system. We make a detailed explanation of its array structure, working principle, and hardware system. However, it is usually difficult to synthesize flexible beams because the phase value is very limited in this antenna. Two methods are proposed in this paper to demonstrate the beam synthesis based on repetitive coding and convolution, and the distribution of beam pointings is analysed on the basis of this mechanism. Experiments that measure the radiation pattern of this antenna are carried out to verify the simulated results using only 1-bit coding metasurface in a radar system, whose phase value is controlled by pin diode on the surface.
\end{abstract}

\section{Introduction}

Phased array radar is extensively adopted into detection in virtue of its flexible properties in beam scanning. Its phase shift properties are constrained by the properties of the phase shifter, which can be easily affected by the temperature and the nature of the device itself. Conventional phased array radar is generally bulky and complex in structure. Although it overcomes the limitations of mechanical scanning for radar performance, there are some new techniques such as printed antenna, conformal array, solid T/R components, time delay device, optical and digital beamforming being applied these days. The huge cost of T/R components, large size, weight, and manufacturability always push the innovation and progress of the whole technology. The metamaterial particles and digital codes are incorporated with each other in the wake of the proposed coding metamaterial, which have aroused wide concern recently [1-3]. Based on coding metamaterial, it can realize the real-time control of EM wave, which is directly related to the information domain to physical domain $[4,5]$.

In 2013, Lipworth et al. in Duke University proposed that aperture made by metamaterial could be used to realize compressive microwave imaging, and the whole system would require only a microwave detector $[6,7]$. Resonance units with different resonant frequencies are randomly distributed on metamaterial surfaces [8]. With the change of the frequency of the excitation source, the radiation pattern of the antenna has been changed and many different measurement modes can be obtained by frequency scanning in the operating bandwidth.

The research of artificial electromagnetic material was carried out in the National Key Laboratory of Millimetre Wave in the Southeast University in China several years ago. Their team has designed a series of metasurfaces with ultralow profiles and used them in the fields of beam synthesis, microwave imaging, and holographic surfaces [9]. They also proposed a millimetre-wave active imaging method based on the combination of metamaterial and machine learning imaging algorithm. They use the FPGA (field-programmable gate array) to control the working state of the artificial electromagnetic material and use machine learning algorithms to get the high-resolution image of the target. The system requires only a single millimetre-wave transmitter and receiver, so it has extremely low hardware cost. The 
system does not require a mechanical scan, so it can image in real time [10].

In this paper, a radar system with a coding metamaterial antenna is introduced. Its performance in beam synthesis and target detection where the number of coded bits for each element in the coding metasurface may not be substantial subject to hardware conditions. The wavefronts of EM waves can be steered via the discontinued phase introduced at a subwavelength scale superficially on substrates in virtue of this system. The focus of this paper is mainly to analyse how to synthesize flexible beams and to verify it with experiment when a very confined phase value is employed in a metamaterial antenna, which is called "low-bit coding metasurface". For conventional phased array antennas, it can achieve continuous beam synthesis because of its flexible and continuous phase shifters, where the phase difference between the adjacent elements is related to the beam deviation. Another difference is that the phase difference between every adjacent element is always a fixed value in order to obtain the maximum coherence stack of the distant field in the direction of beam synthesis. However, in metamaterial antenna, the phase difference which can be selected is very limited. So, the coding rules can no longer be simply set according to the mapping relationship between the beam pointing and the phase difference. We also set up an experimental system to prove our theory.

The rest of the paper is organized as follows: Section 2 presents the hardware of the antenna system. We demonstrate the mechanism of phase-controlled surface and its working parameters, which is the foundation for the beam synthesis analysis (with very confined phase values) in the following chapters. Then, analytical beam synthesis methods are proposed in Section 3, where we give the equations and numerical simulation of the radiation pattern of low-bit coding material antenna. In Section 4, the concrete experiment and measurement based on the proposed theory are presented to verify the simulation. Finally, conclusions are made in Section 5.

\section{Hardware}

The coding metasurface consists of different subwavelength resonant particles where the phase shift is represented by discrete codes like " 0 " and " 1 ", which can be employed to represent the phase value $0^{\circ}$ and $180^{\circ}$. The surface that we use in this experiment includes $20 \times 20$ elements made from subwavelength resonant particles, and the phase value is controlled by the pin diode. When the pin diode in each element is on, its voltage is $0.8 \mathrm{~V}$, which represents phase value $180^{\circ}$. When it is off, its voltage is $0 \mathrm{~V}$, which represents $0^{\circ}$. When the EM wave emitted by the horn antenna is reflected by the metasurface, its phase changes accordingly. At present, the advanced metamaterial antenna can realize phase modulation by up to 2-bit code, which has two layers in a unit cell on the metasurface. There is a pin diode on each layer whose states (on or off) can represent 4 phase values $\left(00,01,10\right.$, and 11 , or $0^{\circ}, 90^{\circ}, 180^{\circ}$, and $270^{\circ}$, resp.). It means that the codes are not represented by effective medium parameters and the metasurface can be digitally controlled to produce different radiation patterns, which is realized by hardware such as FPGA. So, the binary codes can change the diode states on the unit cells and result in the change of the transmission characteristics of the entire coding metasurface [11].

The feed in this antenna is not realized by a feed network but by a horn antenna (shown in Figure 1), which means that the antenna surface is illuminated by the horn antenna. There is a code input port on the side. When the power switch is on, the RF signals are fed into the horn antenna and the coding scheme calculated previously is input via the code input port, which provides the phase values in parallel for each element on the antenna and controls the beam pointing in real time. The feeder transmits the fixed power to the horn antenna.

A schematic diagram of this detection system is presented in Figure 2. DSP component provides the specific signal parameters such as pulse width (100 ns), bandwidth $(200 \mathrm{MHz})$, pulse repetition interval (PRI, 10 $\mathrm{s}$ ), and carrier frequency $(500 \mathrm{MHz})$. The intermediate frequency (IF) signal whose frequency range is from $400 \mathrm{MHz}$ to $600 \mathrm{MHz}$ is upconverted twice $(2.4 \mathrm{GHz}$ and $6.6 \mathrm{GHz}$, resp.) in the $\mathrm{T} / \mathrm{R}$ components. So, the frequency range of the output signal from $\mathrm{T} / \mathrm{R}$ is from 9.4 to $9.6 \mathrm{GHz}$. Then, the signal is transmitted to the horn antenna and then illuminates the reflective metasurface. Another path in this system is the code input, which starts from DSP and to the small FPGA component in the metamaterial antenna. The code scheme is designed based on the parameters of the antenna and signal based on the experimental demand, and the code is input directly during the experiment to realize the beam control.

\section{Beam Synthesis}

3.1. Repetitive Coding. For a uniform line array antenna, in the case of all the elements equally coupled with amplitude $E$, the radiation pattern of far field is defined as

$$
E(\theta)=E \sum_{k=0}^{N-1} e^{j k(\psi-\varphi)},
$$

where $\lambda, \theta, \theta_{0}, N$, and $d$ denote the wavelength, the angle deviated from the normal, the beam pointing, the amount of elements, and array spacing, respectively. $\psi=(2 \pi / \lambda) d$ $\sin \theta$ refers to the phase difference resulting from the difference in wave path, and $\varphi=(2 \pi / \lambda) d \sin \theta_{0}$ denotes the difference in excitation current phase produced by adjacent elements. So, the range of the beam scan is affected by the range of $\varphi$, which suggests that there are only 4 beam pointings when it is 2-bit coding and only 2 beam pointing when it is 1-bit coding. And because the wavelength cannot be changed randomly, it makes sense to change the array spacing $d$.

Arising from the confined phase values in the foregoing system, each code shall be $p$ times repeated to elevate the "equivalent array spacing" $d$ and $d=p d_{0}$. $d_{0}$ denotes the 




FIGURE 1: The experimental setup in a microwave anechoic chamber.

actual physical spacing on the metasurface. For this reason, the beam pointing shall be determined by

$$
\sin \theta_{0}=\frac{\lambda}{p d_{0} 2^{M}}
$$

where $M$ is the code bit.
The simulation shows that this method is feasible (see Figure 3). The coding matrix refers to the arrangement state of the encoding value on the metasurface containing $100 \times 100$ elements, where the color in each element represents its corresponding values. For 2-bit coding in Figure $3(\mathrm{a})$, its phase values $0^{\circ}, 90^{\circ}, 180^{\circ}$, and $270^{\circ}$ are represented by $00,01,10$, and 11 , respectively, which can also be denoted by $0,1,2$, and 3 in the color bar. To explain this process better, let $\lambda=d_{0} \times 2^{M}$, so the beam pointing is $\theta_{0}=\arcsin (1 / p)$.

To prove that the repetition of the code is indeed equivalent to increase the "equivalent spacing" of the element, the analytic and approximate radiation pattern are both given for comparison. The analytic radiation expression refers to the actual pattern by repetitive coding, and the approximate expression refers to the result got by replacing $d$ with $p d_{0}$ in the radiation pattern of the conventional phased array.

For the analytic radiation expression, the codes are generally arranged in the following order (shown in Table 1) in a uniform line array.

To facilitate the calculation, the element numbers who have the same phase are put into the same matrix. $\boldsymbol{\beta}_{\mathbf{i}}\left(i=0,1, \ldots, 2^{M}-1\right)$ contains the element numbers whose phase is $2 \pi i / 2^{M}$. On that basis, in line with the order of arrangement exhibited in Table 1 , it is able to be defined as

$$
\boldsymbol{\beta}_{\mathbf{i}}=\left[\begin{array}{cccc}
p \times i & p \times i+1 & \cdots & p \times i+p-1 \\
p \times\left(2^{M}+i\right) & p \times\left(2^{M}+i\right)+1 & \cdots & p \times\left(2^{M}+i\right)+(p-1) \\
\vdots & \vdots & \ddots & \vdots \\
p \times\left[2^{M} \times(l-1)+i\right] & p \times\left[2^{M} \times(l-1)+i\right]+1 & \cdots & p \times\left[2^{M} \times(l-1)+i\right]+(p-1)
\end{array}\right] .
$$

Assuming that the amount of elements is exactly $N=2^{M} \times p \times l$, the analytic radiation pattern can be acquired as follows:

$$
E(\theta)=\frac{1-e^{j \omega p}}{1-e^{j \omega}} \times \frac{1-e^{j \omega p l \times 2^{M}}}{1-e^{j \omega p \times 2^{M}}} \times \sum_{k=0}^{2^{M}-1} e^{-j\left(2 \pi k / 2^{M}-k p \omega\right)}
$$

where $\omega=\left(2 \pi d_{0} / \lambda\right) \sin \theta$.

For approximate expression, simply use $p d_{0}$ to replace $d$ in conventional phased array antenna radiation pattern:

$$
|E(\theta)| \approx \frac{\sin \left[(N / 2)\left(\left(2 \pi p d_{0} / \lambda\right) \sin \theta-2 \pi / 2^{M}\right)\right]}{\sin \left[1 / 2\left(\left(2 \pi p d_{0} / \lambda\right) \sin \theta-2 \pi / 2^{M}\right)\right]} .
$$

The results are exhibited in Figure 4 with the simulation of the above two methods.
It can be seen from the simulated results that the approximate pattern is highly similar to the actual pattern in the beam pointing although there is a difference between their main lobe width and side lobe level. It provides an important theoretical support for repetitive coding, where it can achieve the similar results of increasing the physical array spacing. Although this method is very straightforward, its synthetic beam pointing is very confined. If the appropriate wavelength, code bits, and other parameters are selected, it may produce $\sin \theta_{0}=1 / 2 p, \sin \theta_{0}=1 / p, \sin \theta_{0}=2 / p, \sin \theta_{0}=3 / p$, and so on. The distribution of beam pointing is indicated in Figure 5.

Arising from the properties of the inverse trigonometric function itself, the beam pointing is centrally distributed at small angles $\left(\theta_{0}<15^{\circ}\right)$ in any case to adjust to other parameters. In practical applications, wavelength and code bits cannot be changed once determined in a real radar system made by the metasurface. It is of great necessity to ascertain others methods of synthesizing more flexible beams. 




FIgURE 2: A schematic diagram of the metamaterial antenna detection system.

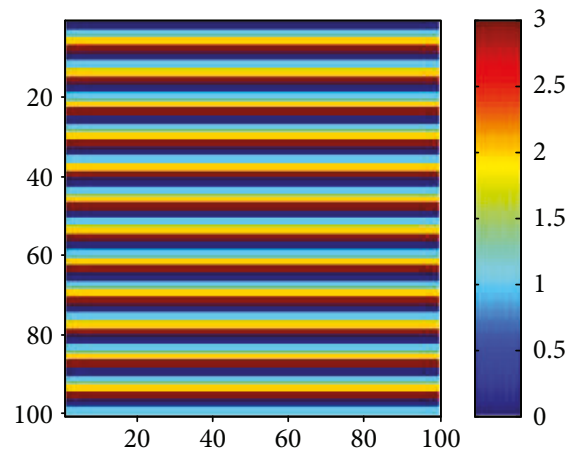

(a)

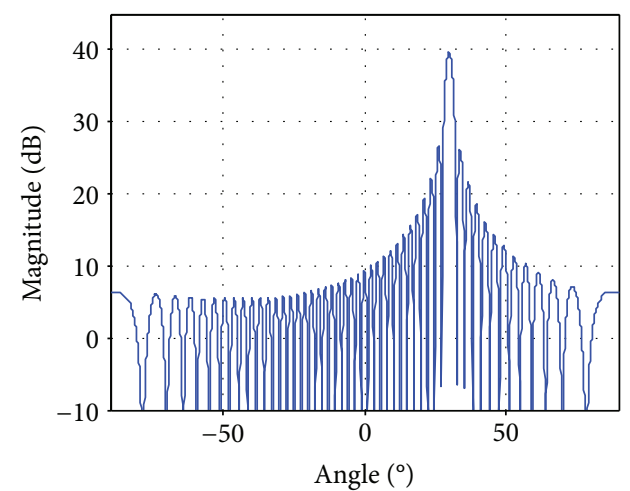

(b)

FIGURE 3: Simulated results when $\lambda=d_{0} \times 2^{M}, d_{0}=1 \mathrm{~cm}, N=100, M=2$, and $p=2$. (a) Coding matrix. (b) Radiation pattern: beam pointing: $29.98^{\circ} \approx \arcsin (1 / 2) ; 3 \mathrm{~dB}$ width: $2.41^{\circ}$; side lobe level: $-13.10 \mathrm{~dB}$.

TABLE 1: Coding arrangement in a uniform line array.

\begin{tabular}{|c|c|c|c|c|c|c|c|c|c|c|c|}
\hline \multicolumn{12}{|l|}{ Uniform line array } \\
\hline Sum & \multicolumn{11}{|c|}{$2^{M} \times p \times l$} \\
\hline Sum & & & & & & $2^{M} \times p$ & & & & & $\ldots \ldots$ \\
\hline Code & 0 & $\ldots$ & 0 & 1 & $\ldots$ & 1 & $\ldots$ & $2^{M}-1$ & $\ldots$ & $2^{M}-1$ & $\ldots \ldots$ \\
\hline Element number & 0 & $\ldots \ldots$ & $p-1$ & $\ldots \ldots$ & $\ldots \ldots$ & $2(p-1)$ & $\ldots \ldots$ & $\ldots \ldots$ & $\ldots \ldots$ & $2_{(p-1)}^{M}$ & $\cdots \cdots$ \\
\hline
\end{tabular}

3.2. Beam Synthesis by Convolution. When the repetitive coding method is adopted, the far field radiation pattern can be decomposed from (1) as follows:

$$
E(\theta)=E \sum_{k=0}^{N-1} e^{j k \psi-j \varphi_{k}}
$$

It can be seen from (6) that if $e^{-j \varphi_{k}}$ is regarded as a timedomain sequence with $k$ as a time variable, the radiation pattern $E$ is like the Fourier transform of this time-domain sequence. Through the analysis of the antenna pattern, it can be concluded that its shape is similar to a sinc function. The sinc function has similarities to the impulse function, which has a good convolution shift property. Combined with some of the basic beam points of the repetitive foregoing coding method, it can synthesize flexible beams.
Without loss of generality, the following sequence of time domains shall be written as follows:

$$
f(k)=e^{-j k \varphi}
$$

where $k=0,1, \ldots, N-1$. When $\psi$ is replaced by $-\omega$ in (6), the following equation is proved:

$$
f_{1}(k) f_{2}(k) \Leftrightarrow \frac{1}{2 \pi} F_{1}(\omega) * F_{2}(\omega)
$$

where $*$ denotes the convolution operation. The frequency domain function conforms to the following properties:

$$
\begin{aligned}
F\left(\omega-\omega_{1}\right) * F\left(\omega-\omega_{2}\right)= & {\left[F(\omega) * \delta\left(\omega-\omega_{1}\right)\right] } \\
& *\left[F(\omega) * \delta\left(\omega-\omega_{2}\right)\right] \approx F\left(\omega-\omega_{1}-\omega_{2}\right) .
\end{aligned}
$$






Figure 4: Simulated results when $N=48, l=6, M=2, d_{0}=1 \mathrm{~cm}$, $f=9.5 \mathrm{GHz}$, and $p=2$. (Blue curve) approximate radiation pattern: $29.75^{\circ} \approx \arcsin (1 / 2) ; 3 \mathrm{~dB}$ width: $2.87^{\circ}$; side lobe level: $-13.34 \mathrm{~dB}$. (Red curve) analytic radiation pattern: $29.75^{\circ} \approx \arcsin (1 / 2) ; 3 \mathrm{~dB}$ width: $6.31^{\circ}$; side lobe level: $-12.91 \mathrm{~dB}$.

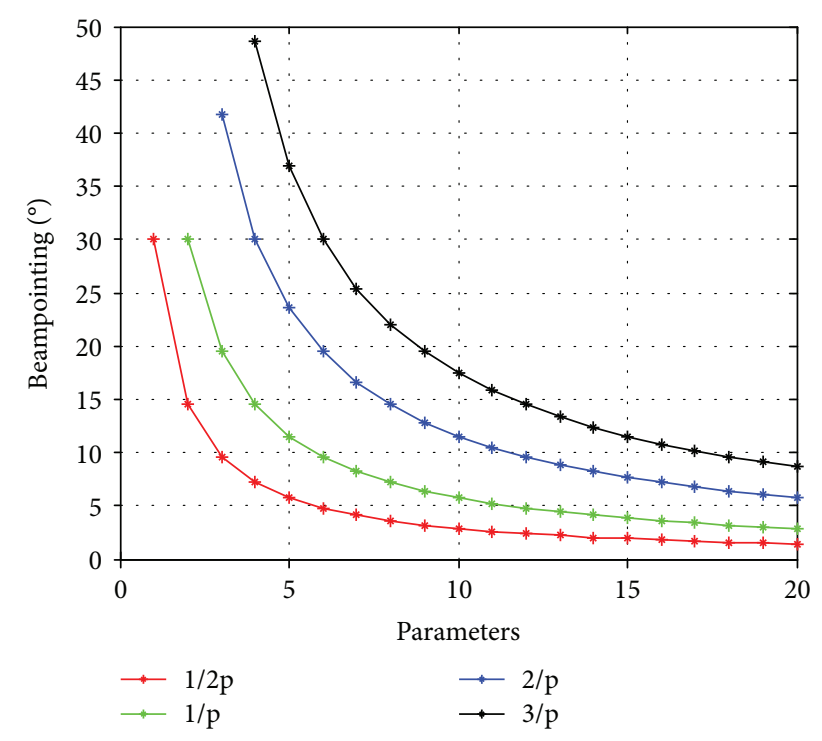

Figure 5: Beam pointing distribution with different parameters.

The approximation adopted above merely denotes the approximation of the beam pointing. When two "time arrays," namely, $f_{1}(k)=e^{-j k \varphi_{1}}$ and $f_{2}(k)=e^{-j k \varphi_{2}}$, are multiplied, their phase arrays $\left(\varphi=\varphi_{1}+\varphi_{2}\right)$ shall be added [12]. Therefore, the synthesized angle shall be analytically expressed as

$$
\sin \theta_{0}=\sin \theta_{1}+\sin \theta_{2}
$$

In that the angle domain is projected to the frequency domain $(-\omega=(2 \pi d / \lambda) \sin \theta)$, the Fourier transform mentioned in this chapter shall be explicated as the spatial Fourier transform.
3.3. 3-D Beam Synthesis. When it comes to 3-D beam synthesis, the metasurface contains $m$ rows and $n$ columns of elements. The row and column spacing are denoted by $d_{1}$ and $d_{2}$, respectively. Through employing the foregoing approximation, 3-D radiation pattern shall be defined as

$$
\begin{aligned}
E(\theta, \varphi)= & \sum_{i=0}^{n-1} \sum_{k=0}^{m-1} \exp \left\{j \left[\left(\frac{2 \pi i d_{1}}{\lambda} \cos \theta \sin \varphi-\eta_{i k}\right)\right.\right. \\
& \left.\left.+\left(\frac{2 \pi i d_{2}}{\lambda} \sin \theta-\zeta_{i k}\right)\right]\right\},
\end{aligned}
$$

where $\theta, \varphi, \eta_{i k}$, and $\xi_{i k}$ are elevation, azimuth, row phase difference, and column phase difference. Using the approximation method in (5), the radiation pattern in horizontal and vertical directions can be got, respectively:

$$
\begin{gathered}
\left|E_{1}(\theta, \phi)\right| \approx \frac{\sin \left[(n / 2)\left(\left(2 \pi p d_{1} / \lambda\right) \cos \theta \sin \varphi-2 \pi / 2^{M}\right)\right]}{\sin \left[1 / 2\left(\left(2 \pi p d_{1} / \lambda\right) \cos \theta \sin \varphi-2 \pi / 2^{M}\right)\right]}, \\
\left|E_{2}(\theta)\right| \approx \frac{\sin \left[(m / 2)\left(\left(2 \pi q d_{2} / \lambda\right) \sin \theta-2 \pi / 2^{M}\right)\right]}{\sin \left[1 / 2\left(\left(2 \pi q d_{2} / \lambda\right) \sin \theta-2 \pi / 2^{M}\right)\right]} .
\end{gathered}
$$

The total coding pattern is the modular addition of sub coding patterns. $\mathbf{p}=\left[p_{1}, p_{2}, \ldots, p_{a}\right]$ means a sub coding patterns which repeat $p_{1}, p_{2}, \ldots, p_{a}$ times to control the azimuth. $\mathbf{q}=\left[q_{1}, q_{2}, \ldots, q_{b}\right]$ means $\mathrm{b}$ sub coding patterns which repeat $q_{1}, q_{2}, \ldots, q_{b}$ times to control the elevation. Then, sum up all the codes under the modular addition $\left(2^{M}\right)$. So, the analytical expressions for the designed elevation and azimuth are as follows:

$$
\begin{aligned}
& \sin \theta_{0}=\frac{\lambda}{d_{2} \times 2^{M}} \sum_{i=1}^{b} \frac{1}{q_{i}}, \\
& \sin \varphi_{0}=\frac{\lambda}{d_{1} \cos \theta \times 2^{M}} \sum_{k=1}^{a} \frac{1}{p_{k}} .
\end{aligned}
$$

To explain this process more vividly, the results of the simulation experiment are given in Figure 6. The predesigned angles are $\theta_{0}=4.78^{\circ}$ and $\varphi_{0}=27.38^{\circ}$, respectively.

To better demonstrate the distribution of the beam pointing synthesized by this method, the following simulation experiment is carried out: assuming that $N=3,1 \leq a, b \leq 4$, and $1 \leq q_{i}, p_{k} \leq 10$, then four different distributions are adopted to model the data, which are normal distribution, Rayleigh distribution, Weibull distribution, and Gamma distribution. The probability density functions (PDF) of four models are shown in Figure 7.

As can be seen from the statistical distribution of the beam, the beam pointings are intensive between $20^{\circ}$ and $50^{\circ}$ while there is almost no distribution under $5^{\circ}$. This is because the number of the repetitions of a single code cannot be too high in the case of a finite number of elements on the metasurface.

In terms of a fixed combination of designed elevation and azimuth, there may be more than one coding matrix. Different performances are indicated in beam pointing accuracy, main 


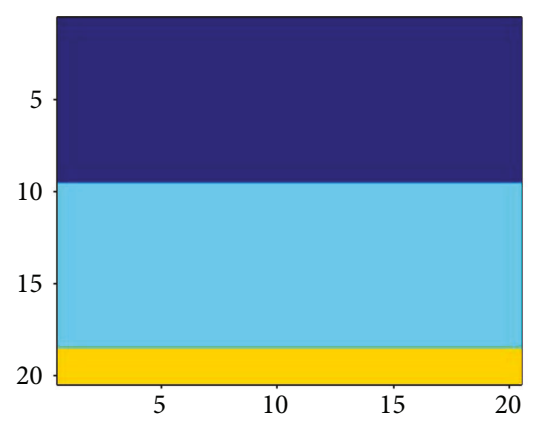

(a1)



(a3)

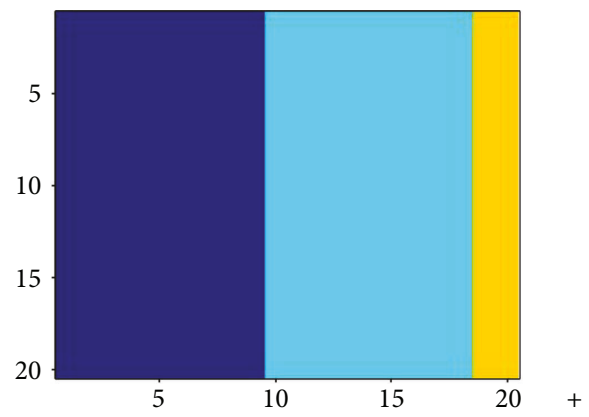

(a2)

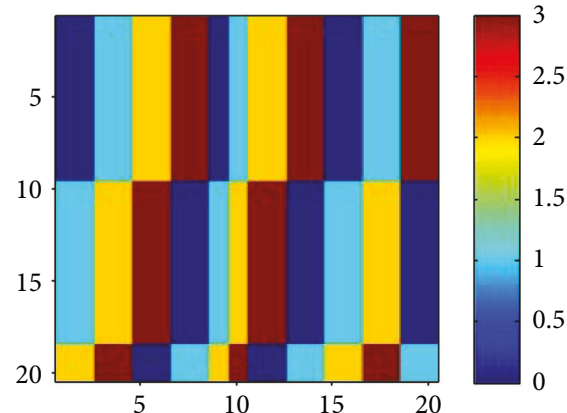

(a)

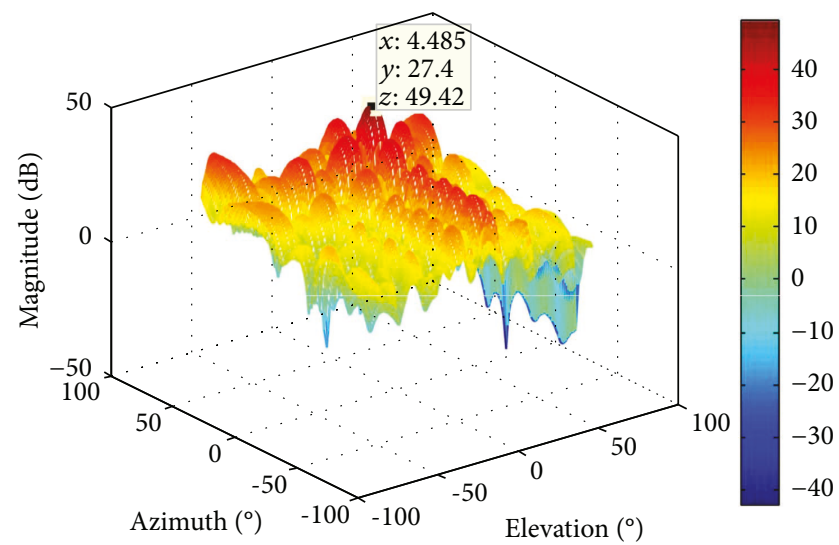

(b)

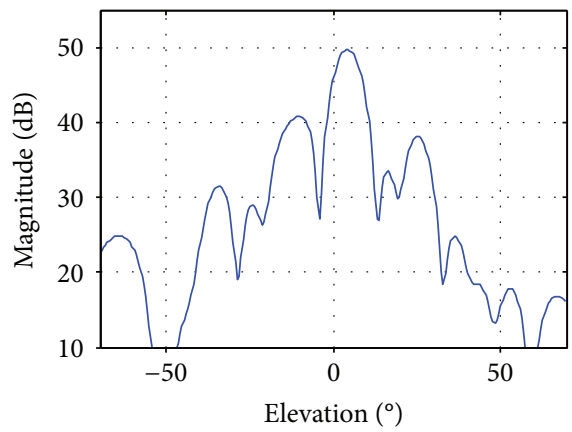

(c)

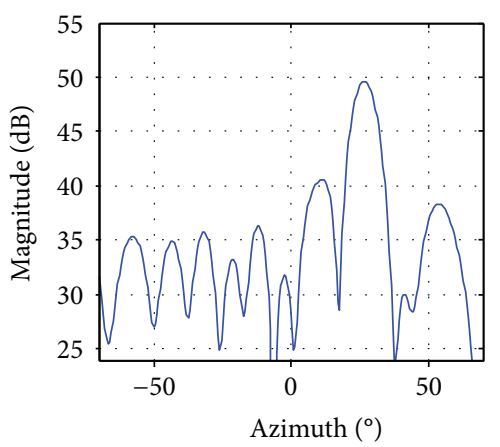

(d)

FIgURE 6: Simulated results of modular addition when $M=2, f=9.5 \mathrm{GHz}, m=n=20, d_{1}=d_{2}=1 \mathrm{~cm}$. (a) The modular addition of the coding value in (a1), (a2), and (a3). The total modular addition is represented by $\mathbf{q}=[9]$ and $\mathbf{p}=[9,2]$. The radiation pattern in (a1) is $\mathbf{q}=[9]$ The radiation pattern in (a2) is $\mathbf{p}=[9]$. The radiation pattern in (a3) is $\mathbf{p}=[2]$. (b) 3-D radiation pattern. (c) Section view when $\varphi=\varphi_{0}$, beam pointing is $4.48^{\circ}, 3 \mathrm{~dB}$ width is $10.29^{\circ}$, and side lobe level is $-8.92 \mathrm{~dB}$. (d) Section view when $\theta=\theta_{0}$, beam pointing is $26.83^{\circ}, 3 \mathrm{~dB}$ width is $11.76^{\circ}$, and side lobe level is $-9.04 \mathrm{~dB}$. 


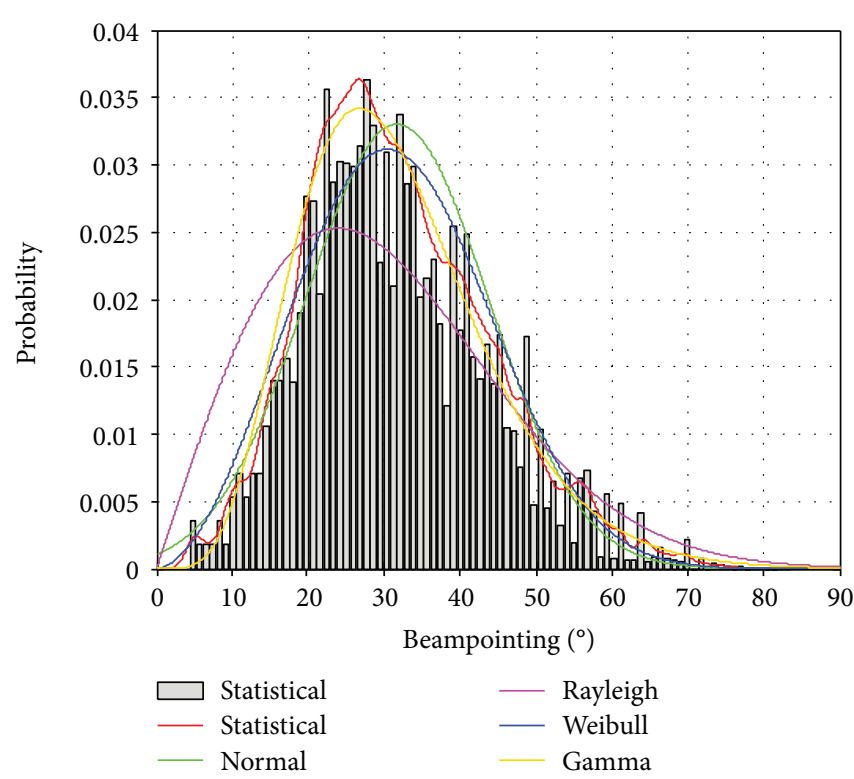

(a)

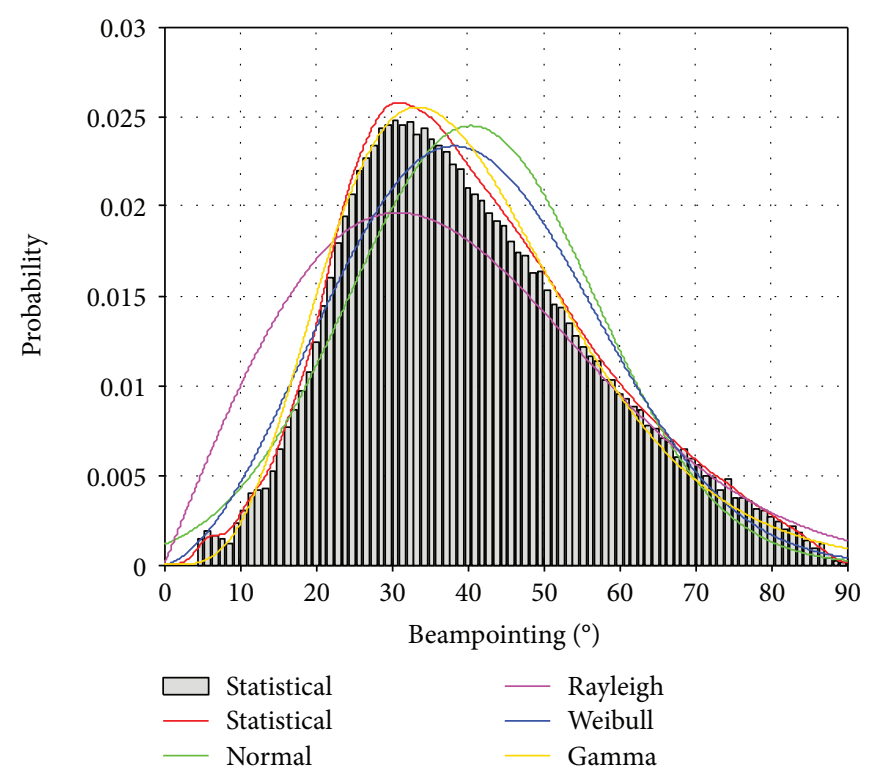

(b)

FIgure 7: Statistical result and distribution models of the beam pointing when $M=2, f=9.5 \mathrm{GHz}, m=n=20$, and $d_{1}=d_{2}=1 \mathrm{~cm}$. (a) For elevation and (b) for azimuth.



(a)

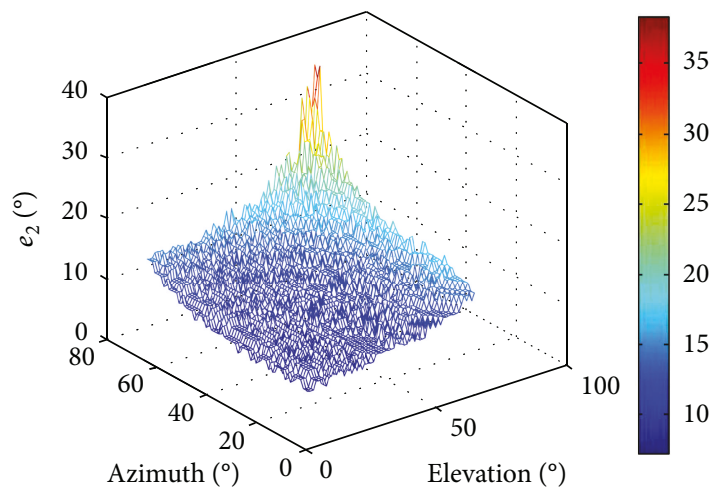

(b)

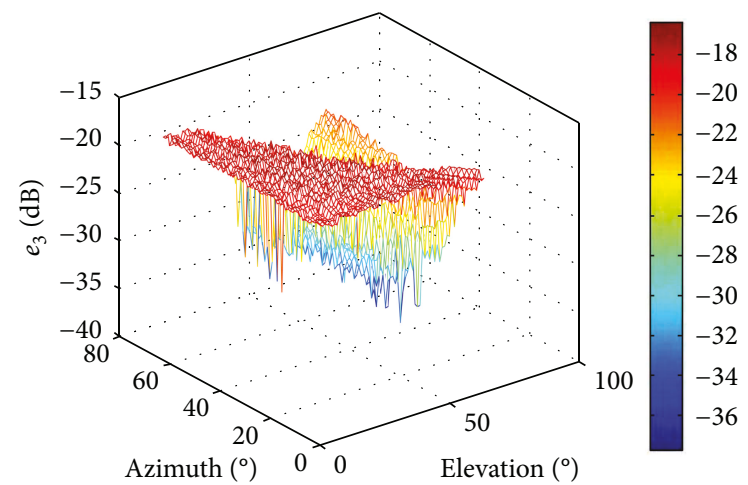

(c)

Figure 8: The distribution of cost function for (a) beam pointing error, (b) main lobe width cost, and (c) side lobe level cost. 




(a)



(b)

Figure 9: The comparison (section view and normalized magnitude) of simulated and experimental results when elevation is $0^{\circ}$. (a) Azimuth is $30^{\circ}$. (b) Azimuth is $42^{\circ}$.

lobe width, and side lobe level. To seek out the optimal coding matrix, the normalized errors or costs of them shall be overall reckoned with to comprise the following cost function:

$$
c=\alpha\left(e_{1 e}^{2}+e_{1 a}^{2}\right)+\beta\left(e_{2 e}^{2}+e_{2 a}^{2}\right)+\gamma\left(e_{3 e}^{2}+e_{3 a}^{2}\right),
$$

where $c$ is the total cost and $e_{1}, e_{2}$, and $e_{3}$ are normalized cost for beam pointing accuracy, main lobe width, and side lobe level, respectively. The subscripts $e$ and $a$ are for elevation and azimuth, respectively. The distribution of cost functions is shown in Figure 8.

\section{Experimental Results and Discussions}

The experiment was carried out in a microwave anechoic chamber using the metamaterial antenna in the radar system described in Figure 2. The metasurface was illuminated by an offset-fed horn antenna to avoid the shielding of the main radiation as shown in Figure 1. The radar system is designed by Southeast University in China.

By using vector network analyzer, we got the radiation pattern and compared it with the simulated results. The simulated and experimental results are compared in Figure 9, which suggest that the main lobe of the experimental results basically conforms to the simulated results. It shows that this coding metasurface has the ability to control the beam pointing, but it has relatively high side lobes and wide main lobe (nearly $10^{\circ}$ ), which may affect the performance of target detection. A finite number of elements leads to the widening of the main lobe while the relative flat feeding mode on the metasurface leads to the increase of the side lobe. It is also clear from the results that insufficient code bits (only 1-bit) in this antenna leads to the presence of two symmetrical main lobes. This can be improved if 2-bit coding is implemented, but it cannot be realized due to the limitation of hardware conditions in this experiment. Another issue is the quality of the antenna itself, which means the welding and package level influence and the performance of the antenna.

\section{Conclusions}

In this paper, we propose a metamaterial antenna with 400 elements on the surface and the phase values are controlled by the pin diodes. It is made without a very large feed network but by only a horn antenna, which illuminates the coding surface and makes the antenna more miniaturized and flexible. We present the beam synthesis methods with regard to this kind of antenna, where the code bits are limited to 1 or 2 bit. Through employing repetitive coding and convolution properties, the number of beam pointing that can be synthesized on the metasurface is greatly increased. We analyze the distributions of the predesigned beam pointings produced by these two methods. This not only achieves beam synthesis using metamaterial antennas but also provides an approach to realize beam synthesis under special conditions where phase values are confined in the conventional phased array antenna. We also use the experiment to verify this theory of beam synthesis by taking two azimuths for the test. As the result shows, although the amplitude is different at every point, the position of the main and side lobe is basically accurate. There are two main problems to be solved in the future work: First, beam synthesis under about $5^{\circ}$ (deviation from the normal of the metasurface) is difficult for the two proposed methods since it needs large repetition times $(p)$. This is unrealistic in the case of a finite number of array elements. Second, optimization of the radiation pattern needs to be implemented because it is clear that the side lobe level is very high for both experimental and simulated results. Side lobe suppression is mainly achieved by changing the distribution of radiation field on 
the metasurface, so the position, power, and radiation pattern of the horn antenna need to be further designed.

\section{Conflicts of Interest}

The authors declare that there are no conflicts of interest regarding the publication of this paper.

\section{Acknowledgments}

The paper is supported by the National Natural Science Foundation of China under Grant 61571011.

\section{References}

[1] J. Hunt, T. Driscoll, A. Mrozack et al., "Metamaterial apertures for computational imaging," Science, vol. 339, no. 6117, pp. 310-313, 2013.

[2] M. El Badawe, T. S. Almoneef, and O. M. Ramahi, "A true metasurface antenna," Scientific Reports, vol. 6, no. 1, 2016.

[3] T. J. Cui, D. Smith, and R. Liu, Metamaterials: Theory, Design, and Applications, Springer Publishing Company, Incorporated, 2009.

[4] R. Liu, C. Ji, T. Cui, and D. R. Smith, "Progress of metamaterials at microwave frequencies," International Workshop on Metamaterials, pp. 47-49, 2008.

[5] T. J. Cui, "Microwave metamaterials - from passive to digital and programmable controls of electromagnetic waves," Journal of Optics, vol. 19, no. 8, p. 084004, 2017.

[6] G. Lipworth, J. Hunt, A. Mrozack, D. Brady, and D. R. Smith, "Simulations of 2D metamaterial apertures for coherent computational imaging," in 2013 IEEE International Conference on Microwaves, Communications, Antennas and Electronic Systems (COMCAS 2013), vol. 1544no. 6, pp. 1-4, Tel Aviv, Israel, October 2013.

[7] G. Lipworth, A. Mrozack, J. Hunt et al., "Metamaterial apertures for coherent computational imaging on the physical layer," Journal of the Optical Society of America A, vol. 30, no. 8, p. 1603, 2013.

[8] C. M. Watts, D. Shrekenhamer, J. Montoya et al., "Terahertz compressive imaging with metamaterial spatial light modulators," Nature Photonics, vol. 8, no. 8, pp. 605-609, 2014.

[9] T. J. Cui, S. Liu, and L. L. Li, "Information entropy of coding metasurface," Light: Science \& Applications, vol. 5, no. 11, article e16172, 2016.

[10] Q. Zhang, X. Wan, S. Liu, J. Y. Yin, L. Zhang, and T. J. Cui, "Shaping electromagnetic waves using software-automaticallydesigned metasurfaces," Scientific Reports, vol. 7, no. 1, p. 3588, 2017.

[11] Y. B. Li, L. L. Li, B. B. Xu et al., "Transmission-type 2-bit programmable metasurface for single-sensor and singlefrequency microwave imaging," Scientific Reports, vol. 6, no. 1, article 23731, 2016.

[12] S. Liu, T. J. Cui, L. Zhang et al., "Convolution operations on coding metasurface to reach flexible and continuous controls of terahertz beams," Advanced Science, vol. 3, no. 10, article 1600156, 2016. 




\section{Enfincering}
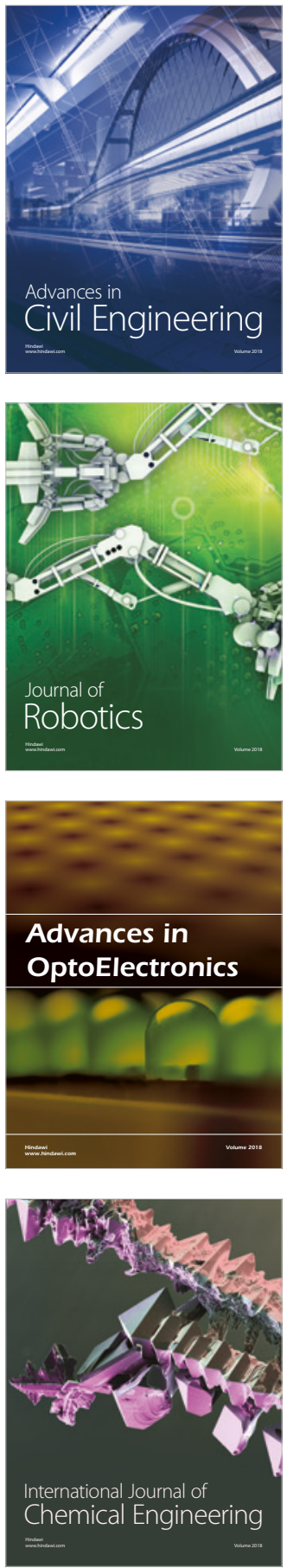

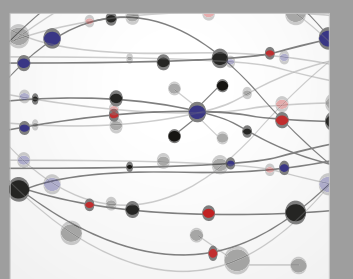

\section{Rotating \\ Machinery}

The Scientific World Journal



Submit your manuscripts at

www.hindawi.com

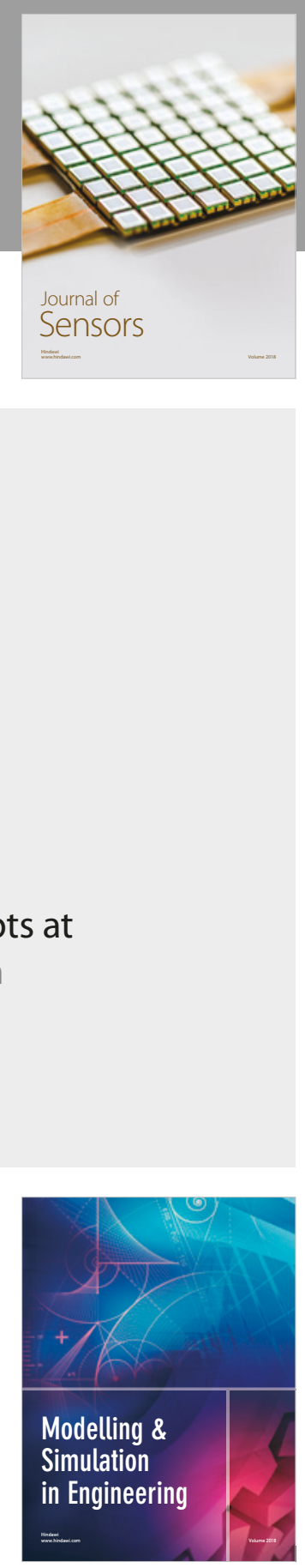

\section{Advances \\ Multimedia}
